Submitted to:

\title{
Design of a Resonant Extraction System for the AGS Booster *
}

\author{
$\underline{K}^{\text {B. Brown }}{ }^{\dagger}$, J. Cullen, J.W. Glenn, Y.Y. Lee, A. McNerney, J. Niederer, T. Roser, A. Soukas, \\ J. Tuozzolo, N. Tsoupas, AGS Dept., BNL, Upton, NY,
}

\begin{abstract}
The Booster Application Facility (BAF) will employ heavy ion beams of many different ion species and at beam energies ranging from 0.04 to $3.07 \mathrm{GeV} /$ nucleon. Resonant extraction is required in order to deliver a continuous stream of particles. In this report we describe the beam requirements and the system design. The basic design is a third integer resonant extraction process which employs a single thin magnetic septum and a thick septum ejector magnet. The expected extraction efficiency is about $85 \%$, based on the thin septum thickness and the predicted step size of the resonant beam at the septum. This is more than sufficient for the low intensity low energy heavy ion beams needed for the BAF. In this report we will present a detailed discussion of the design of the various elements and a discussion of the detailed modeling of resonant extraction from the AGS Booster. The extraction process was modeled using a BNL version of MAD which allowed us to interactively observe detailed particle tracking of the process. This was a key tool to have in hand which permitted us to pose and answer various questions in a very short period of time.
\end{abstract}

\section{INTRODUCTION}

The AGS Booster has operated since 1991 as an injector of protons and heavy ions into the AGS. The operating parameters are summarized in table 1 . The design of the resonant extraction system from the Booster for BAF requires some rearrangement of existing apparatus in the Booster. In the Booster D6 straight section (2.6 $\mathrm{m}$ in length), we will be removing the Booster Beam dump and a wall current monitor. These will be relocated in the Booster B6 straight section. The D6 straight section will then contain a new thick septum magnet, a stripping foil and flag (for beam profiles) mechanism. In addition we will modify the quarter cell vacuum chambers. In the D3 straight section we will remove the current beam dump kicker magnet (which will not be relocated), move an ionization profile monitor instrument to the upstream end of the section, and install a new thin septum magnet. The D4 half cell will be modified for a larger aperture quadrupole vacuum chamber. In addition four horizontal sextupoles will have new power supplies so that they may be used to create a sextupole resonance, as well as track the normal chromaticity system. Finally, the existing backleg windings in the Booster Dipole magnets

\footnotetext{
- Work supported by the U.S. Department of Energy.

$\dagger$ Email: kbrown@bnl.gov
}

D1, D2, D7 and D8 will be connected to provide local orbit bump control at D3 and D6. The D3 thin septum magnet will be movable over a small range, to allow maximizing the extraction efficiency for a given resonance step size.

Table 1: AGS Booster Parameters

\begin{tabular}{l|c}
\hline Parameter & Value \\
\hline Circumference & $201.78(1 / 4 \mathrm{AGS}) \mathrm{m}$ \\
Ave. Radius & $32.114 \mathrm{~m}$ \\
Magnetic Bend R & $13.75099 \mathrm{~m}$ \\
Lattice Type & Seperated Function, FODO \\
No. Superperiods & 6 \\
No. of Cells & 24 \\
Betatron Tunes, X, $\mathrm{Y}$ & $4.82,4.83$ \\
Vacuum Chamber & $70 \times 152 \mathrm{~mm}$ Dipoles \\
& $152 \mathrm{~mm}$ (circular) Quads \\
Max. Rigidity & $17 \mathrm{Tm}$ \\
Injection Rigidity & $2.2 \mathrm{Tm}(200 \mathrm{MeV}$ protons) \\
& $0.9 \mathrm{Tm}(1 \mathrm{MeV} / \mathrm{nuc} \mathrm{Au}(32+))$ \\
Acceleration Rate & $8.9 \mathrm{~T} / \mathrm{s} \mathrm{up} \mathrm{to} 7.5 \mathrm{Tm}(7.5 \mathrm{~Hz})$ \\
& $1 \mathrm{~T} / \mathrm{s} \mathrm{up} \mathrm{to} 17 \mathrm{Tm}(0.7 \mathrm{~Hz})$ \\
\hline
\end{tabular}

The maximum kinetic energy of the extracted beams corresponds to the maximum Booster rigidity of $17 \mathrm{Tm}$ for the heavier ions, which are fully stripped at the stripping foil located at the entrance of the D6 septum magnet, and 13 $\mathrm{Tm}$ for lighter ions. The maximum rigidity of the BAF transport line is $13 \mathrm{Tm}$. Lower energies are possible at lower intensity. Intensities as low as $10^{6}$ ions per pulse will be available to experimenters through collimation just after the D6 septum magnet. Table 1 summarizes the operating parameters for Booster BAF operation.

Table 2: SEB Operating Parameters for Typical Ions

\begin{tabular}{|l|c|c|c|}
\hline Ion & $\begin{array}{c}\text { Charge } \\
\text { in Booster }\end{array}$ & $\begin{array}{c}\text { K.E. Range } \\
\text { (GeV/nucl. })\end{array}$ & $\begin{array}{c}\text { Est. Inten. } \\
{\left[10^{9} \text { Ions/pulse }\right]}\end{array}$ \\
\hline $\mathrm{p}$ & 1 & $0.73 \ldots 3.07$ & 100 \\
$28 \mathrm{Si}$ & 14 & $0.09 \ldots 1.23$ & 4 \\
$56 \mathrm{Fe}$ & 21 & $0.10 \ldots 1.10$ & 0.4 \\
$63 \mathrm{Cu}$ & 22 & $0.10 \ldots 1.04$ & 1 \\
$197 \mathrm{Au}$ & 32 & $0.04 \ldots 0.30$ & 2 \\
\hline
\end{tabular}

\section{BOOSTER RESONANT EXTRACTION}

The main challenge in our design is to have a flexible extraction system, capable of extracting ions uniformly for a duration of several hundred to one thousand milliseconds, without significant impact on the existing subsys- 
tems, including the single turn fast extraction system for beam transfer to the AGS.

The Booster consists of six superperiods and each superperiod has two long open or missing dipole straight sections. The machine will be modified in order to place the extraction magnet at D6. This permits the construction of the experimental area in one of the few available spaces outside the Booster.

Slow extraction is achieved by the controlled excitation of a non-linear betatron third integer resonance. A slow spill is controlled by steering the beam slowly so that particles with different momenta move progressively onto the resonance. Third integer resonant extraction has been used at the AGS for many years for the high energy and heavy ion physics programs, as well as for the radiobiology program. The AGS system is efficient and reliable with a typical extraction efficiency over $96 \%$. [1, 2]

The Booster extraction system will make use of the horizontal third integer resonance at the tune of $Q_{h}=13 / 3$. This tune is significantly lower than the nominal Booster tune, as shown in table 1 . This is required because the Booster tune quadrupoles do not have enough strength to maintain the horizontal betatron tune at the value of $14 / 3$ at the highest energies. To reach even $13 / 3$ at $12 \mathrm{Tm}$ a new power supply for the tume quadrupoles is needed. The required crossing of the half integer resonance at $Q_{h}=$ 9/2 during acceleration is accomplished easily with the fast slew rate available from the tune quadrupole systems, which has been experimentally verified. [3]

A third integer resonance is excited by the $13^{\text {th }}$ harmonic of two sextupole pairs located at C8, F8, B4, and E4. We plan to utilize four of the existing 24 horizontal lattice sextupoles as drive sextupoles. The maximum field of the existing sextupoles is sufficient for their use as drive sextupoles. At the resonance, a stable triangular region of the horizontal phase space is defined within three linear separatrices and the area and orientation of this region can be controlled by the drive sextupoles. In the vicinity of the resonance there is a small range of tunes over which the separatrix degenerates into three narrow legs which have a phase advance of $2 / 3 \pi$ radians with respect to each other. A particle leaving the stable area within the separatrix moves out along one of these legs as it spirals out of the machine. This particle will move from one leg to the next every turn, each revolution stepping further out along the separatrix legs. (see figure 1).

The extraction efficiency depends on the thickness of the first septum as compared to the growth of the resonant betatron amplitude in the final few turns before extraction. So the first septum is made as thin as possible and is aligned precisely so that the maximum growth per turn can be obtained for the available aperture and for the given beam emittance. The step size of a particle at the septum depends on its momentum, betatron amplitude and its proximity to the resonant tune value as well as the drive sextupole strength.

The thin septum is located in the D3 straight section of the Booster. The phase advance to the D6 thick septum is about 71 degrees. An angular kick from the thin septum translates into a large displacement at D6. This can be seen more clearly in figures 1 and 2 , which show the phase space separatrices and the extracted beam trajectories at the thin septum and the thick septum.

The thin septum has an effective thickness of $0.76 \mathrm{~mm}$ and maximum kick of $3 \mathrm{mrad}$, for a length of $1 \mathrm{~m}$. The thick septum has an effective thickness of $15.2 \mathrm{~mm}$, is operated in a dc mode, and gives a kick of $155 \mathrm{mrad}$, for a length of $2.5 \mathrm{~m}$. The drive sextupoles have a maximum normalized strength of $K_{s}=B_{2} \cdot L / B \rho=0.29 m^{-2}$ where $L=0.1 \mathrm{~m}$.

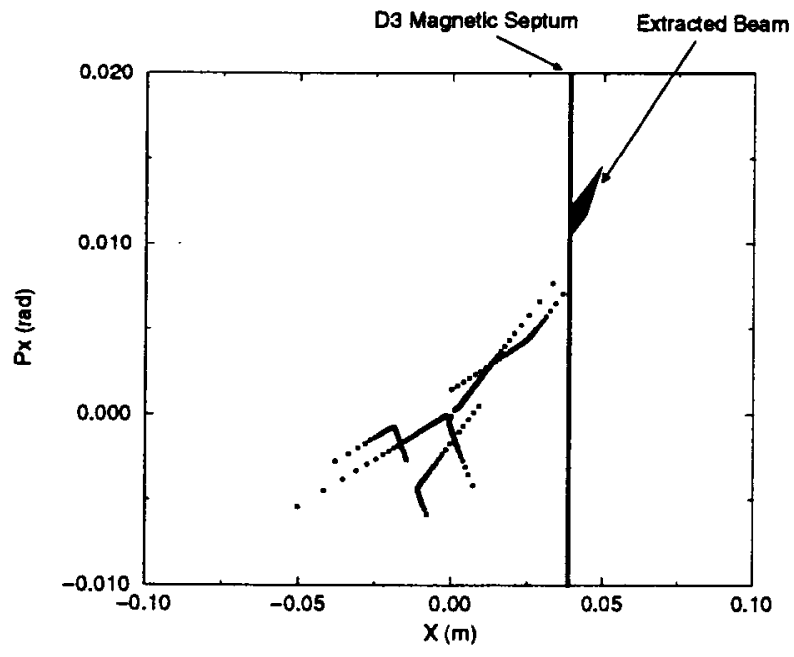

Figure 1: Simulation of resonant beam at the D3 thin septum

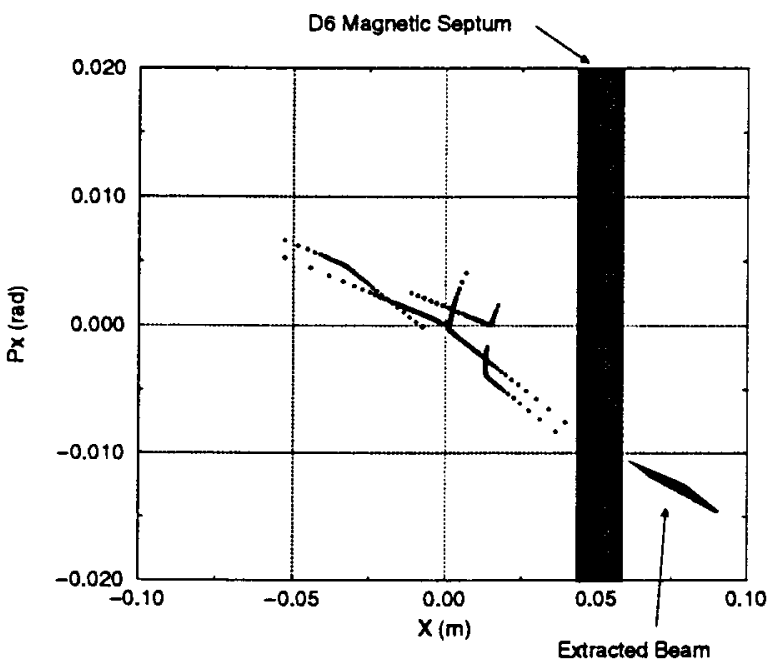

Figure 2: Simulation of resonant beam at the D6 thick septum

Figure 1 shows the phase space growth at the thin magnetic septum with the four drive sextupoles energized. The tune is set to $Q_{h}=13 / 3$ for the particle on the closed orbit, and slightly higher for the particle with emittance of $15 \pi$ mm-mrad. The two pairs of sextupoles are powered with opposite sign at a strength of $0.25 \mathrm{~m}^{-2}$. The spiral pitch is 
slightly over $4.0 \mathrm{~mm}$, which would give an extraction efficiency of $85 \%$. Figure 2 similarly shows the phase space at D6, the location of the thick septum. These simulations were done using a $\mathrm{BNL}$ version of $\mathrm{MAD}$, which incorporates interactive graphics. Using the single particle tracking facilities and the interactive graphics we were able to very quickly study and test different extraction schemes.

\section{PARTICLE DISTRIBUTIONS}

The experimenters that will be using the BAF facilities require highly uniform beam distributions over a $10 \mathrm{~cm}$ diameter beam. In order to accomplish this the beam line will incorporate octupole magnets to create a rectangular distribution at the target.[4] In order to model the behavior of the octupoles we needed to clearly understand the phase space of the extracted beam.

The vertical emittance of the extracted beam is unchanged by the extraction process. To first order, the horizontal emittance is zero, as the phase space area of the extracted beam is completely determined by the momentum spread. A significant amount of dispersion is generated by the extraction process. The extracted beam passes through a stripping foil before entering the D6 thick septum magnet. The thickness of this foil can be adjusted to not only completely strip the ions, but also to provide a large enough horizontal emittance to allow for a uniform spot at the target area. From simulations we determined the beam parameters as shown in table 3 . The horizontal emittance is given as $3 \pi \mathrm{mm} \mathrm{mrad}$, which would require a $0.08 \mathrm{~mm}$ thick copper stripping foil for a $1 \mathrm{GeV} /$ nucleon Fe beam.

Table 3: Extracted Beam Characteristics

\begin{tabular}{|l|c|c|}
\hline & Horizontal & Vertical \\
\hline Emittance & $3 \pi \mathrm{mm}$ mrad & $15 \pi \mathrm{mm} \mathrm{mrad}$ \\
Beta & $10.0 \mathrm{~m}$ & $4.4 \mathrm{~m}$ \\
Alpha & 1.87 & -0.67 \\
$D_{x}$ & $30 \mathrm{~m}$ & $0 \mathrm{~m}$ \\
$D_{x}^{\prime}$ & -3.4 & 0.0 \\
\hline
\end{tabular}

To further understand the behavior of the beam down the BAF line, we performed simulations of 100000 particles, to study beam losses and the uniformity of the beam on target. To do tracking simulations with large numbers of particles is not easily done with a modeling program such as MAD, so we developed a simple program to track many particles, in order to provide a set of input particles for the beam line simulations, and to get a picture of the beam distributions of the beam entering the septum magnets. Figure 3 shows the beam distributions of the extracted beam in the D3 and D6 septums, before any stripping foils. Even before any stripping the beam distributions appear to be Gaussian, even though initially the distribution is rectangular. This is an important result, since the octupoles are most effective at creating a uniform beam distribution when the input beam is Gaussian. [4]

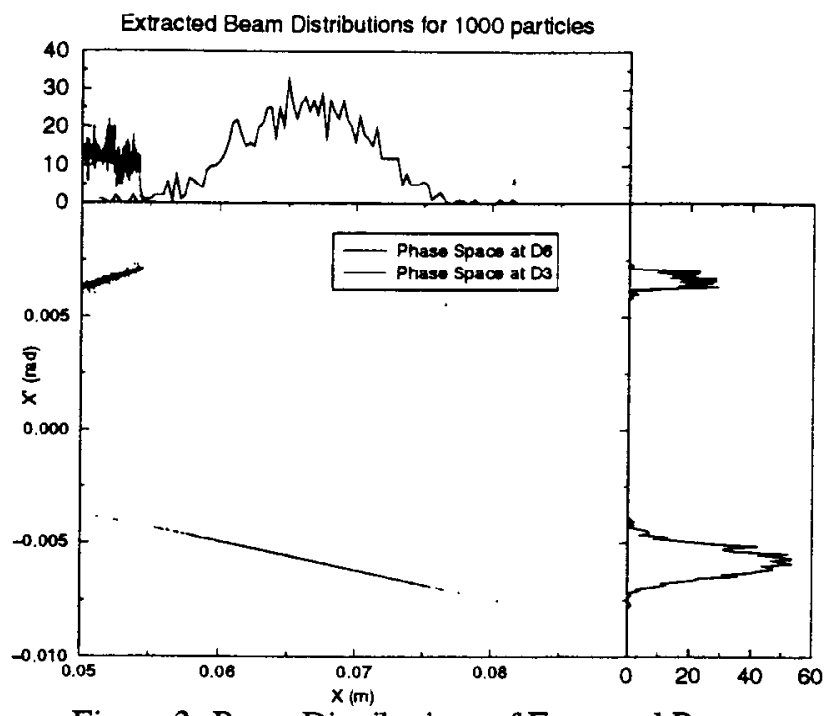

Figure 3: Beam Distributions of Extracted Beam

\section{SPILL STRUCTURE}

To achieve a constant stable output beam intensity, sophisticated control systems are needed in combination with low-ripple power supplies for the main magnets and the extraction magnets. A closed-loop servo will be required which is gated on at the beginning of the flattop. A measurement of the beam spill is compared to a reference signal and generates a correction output signal that controls the Booster main magnet current on flattop and the low frequency part of the spill (to create a constant current beam). The high frequency control is achieved by reducing the ripple of the power supplies. We are also looking into a high frequency feedback to the tune quadrupoles, which has been used successfully at CERN and BNL. In addition we could incorporate a high frequency RF cavity to smooth out $60 \mathrm{~Hz}$ harmonics, again a technique that has been successfully used at both CERN and BNL.

\section{STATUS}

As of the time of this meeting the BAF project has been approved and is funded by the National Aeronautics and Space Administration. Construction has not yet begun, primarily due to the operations schedule for the Booster and AGS, but will begin this summer. The first commissioning of the facility will occur in mid-2001.

\section{REFERENCES}

[1] H. Weisberg and J.W. Glenn, "Exploitation of Nonlinear Growth of Betatron Oscillations to Obtain Efficient Slow Extraction at the AGS", Nucl. Inst. \& Methos 169, 319(1980).

[2] L.Abrens et al, "AGS Resonant Extraction with High Intensity Beams", THP140 in these proceedings.

[3] AGS Internal studies report.

[4] N.Tsoupas et al, "The Booster Applications Facility (BAF) Beam Transfer Line of BNL-AGS Booster", these proceedings. 